\title{
Black Holes in an Effective Field Theory Extension of General Relativity
}

\author{
Vitor Cardoso, ${ }^{1,2}$ Masashi Kimura, ${ }^{1}$ Andrea Maselli, ${ }^{1}$ and Leonardo Senatore ${ }^{3}$ \\ ${ }^{1}$ CENTRA, Departamento de Física, Instituto Superior Técnico-IST, Universidade de Lisboa-UL, \\ Avenida Rovisco Pais 1, 1049 Lisboa, Portugal \\ ${ }^{2}$ CERN 1 Esplanade des Particules, Geneva 23, CH-1211, Switzerland \\ ${ }^{3}$ SITP and KIPAC, Department of Physics and SLAC, Stanford University, Stanford, California 94305, USA
}

(Received 29 August 2018; published 20 December 2018)

\begin{abstract}
Effective field theory methods suggest that some rather general extensions of general relativity include, or are mimicked by, certain higher-order curvature corrections, with coupling constants expected to be small but otherwise arbitrary. Thus, the tantalizing prospect to test the fundamental nature of gravity with gravitational-wave observations, in a systematic way, emerges naturally. Here, we build black hole solutions in such a framework and study their main properties. Once rotation is included, we find the first purely gravitational example of geometries without $\mathbb{Z}_{2}$ symmetry. Despite the higher-order operators of the theory, we show that linearized fluctuations of such geometries obey second-order differential equations. We find nonzero tidal Love numbers. We study and compute the quasinormal modes of such geometries. These results are of interest to gravitational-wave science but also potentially relevant for electromagnetic observations of the galactic center or x-ray binaries.
\end{abstract}

DOI: 10.1103/PhysRevLett.121.251105

Introduction.-The gravitational-wave (GW) astronomy era has began. As the sensitivity of our GW detectors increases, so does our ability to realize the potential of the field. From a mapping of compact objects throughout the visible universe, to measurements of the cosmological expansion rate, the opportunities in both astrophysics and fundamental physics are numerous [1]. GWs carry information from regions of spacetime where gravity is "strong," and therefore are genuine probes of one of the most surprising predictions of general relativity (GR): black holes (BHs). In GR, the uniqueness theorems suggest that BHs are all well described by the Kerr geometry. Such geometry is fully determined by two parameters (mass and spin), which also characterize the full multipolar structure of these objects [1-5]. A simple, well-known method to test such simple multipolar structure is by measuring accurately the final stages of relaxation of a perturbed $\mathrm{BH}$, such as the one created after the inspiral and merger of a BH binary [6-8].

There are compelling reasons to test the nature and the geometry of compact objects such as BHs $[9,10]$. In GR, $\mathrm{BHs}$ harbor singularities where the gravitational field becomes unbounded and where quantum effects may be screened from outside observers thanks to the event

Published by the American Physical Society under the terms of the Creative Commons Attribution 4.0 International license. Further distribution of this work must maintain attribution to the author(s) and the published article's title, journal citation, and DOI. horizon. The assertion that all singularities are hidden from us is so remarkable that any observational evidence for or against it is welcome. Furthermore, the complications associated with putting together GR and quantum mechanics near the Planck scale have been challenging thus far. One resolution is provided by string theory, which is nevertheless a remarkable extension to our laws of physics.

It is thus an extremely appealing prospect that $\mathrm{GW}$ astronomy may be able to improve, even if only incrementally, our understanding of the nature of gravity at high energies.

Technically, in the modern way in which physical laws are written, gravity is "extended" by adding to the GR Lagrangian either new massive particles or new terms built out of the Riemann tensor, suppressed by some mass scale. For associated mass scales much larger than a few inverse kilometers (the scale probed by current $\mathrm{GW}$ observations), it is very hard for these observations to provide any observational guidance on the nature of gravity. Thus, there are theoretical prejudices against embarking upon missions set to test extensions of GR at current scales. However, such preconceptions need not be true: extensions of GR at scales that are probed by experiments such as LIGO or VIRGO are theoretically possible, and not excluded by current experiments or observations. They should be probed.

Effective field theory (EFT) is a useful guide in the search for the most general extension to GR, under the following assumptions: the theory should be testable with GW observations; it should be consistent with other experiments, including short distance tests of GR; it should agree 
with widely accepted principles of physics, such as locality, causality and unitarity; it should not involve new light degrees of freedom. Such an EFT, which is unique, was constructed recently, and has appealing features [11]. By studying the signatures of one single Lagrangian, a vast class of theories is covered: indeed, it is guaranteed that any theory satisfying the abovementioned assumptions will lead to the same signatures as the EFT (for some choice of parameters). However, by using EFT, all information is summarized in constraints (or measurements, if one is lucky) of very few coefficients. Finally, in contrast to phenomenological parametrizations, the one offered by EFTs automatically constrains the observational investigation to the space of physically viable theories, and, within this class, offers the optimal general parametrization of the observational signals.

Effective theory.-Consider the effective action [11]

$$
\begin{aligned}
S_{\text {eff }} & =\int d^{4} x \sqrt{-g} 2 M_{\mathrm{pl}}^{2}\left(R-\frac{\mathcal{C}^{2}}{\Lambda^{6}}-\frac{\tilde{\mathcal{C}}^{2}}{\tilde{\Lambda}^{6}}-\frac{\tilde{\mathcal{C}}}{\Lambda_{-}^{6}}\right), \\
\mathcal{C} & \equiv R_{\alpha \beta \gamma \delta} R^{\alpha \beta \gamma \delta}, \quad \tilde{\mathcal{C}} \equiv R_{\alpha \beta \gamma \delta} \tilde{R}^{\alpha \beta \gamma \delta}
\end{aligned}
$$

where the dual tensor $\tilde{R}^{\alpha \beta \gamma \delta} \equiv \epsilon^{\alpha \beta}{ }_{\mu \nu} R^{\mu \nu \gamma \delta}$, and $\epsilon_{0123}=\sqrt{-g}$. The equations of motion, for $R_{\mu \nu}=0$, read

$$
\begin{aligned}
R^{\mu \alpha} & -\frac{1}{2} g^{\mu \alpha} R \\
= & \frac{1}{\Lambda^{6}}\left(8 R^{\mu \nu \alpha \beta} \nabla_{\nu} \nabla_{\beta} \mathcal{C}+\frac{g^{\mu \alpha}}{2} \mathcal{C}^{2}\right) \\
& +\frac{1}{\tilde{\Lambda}^{6}}\left(8 \tilde{R}^{\mu \rho \alpha \nu} \nabla_{\rho} \nabla_{\nu} \tilde{\mathcal{C}}+\frac{1}{2} g^{\mu \alpha} \tilde{\mathcal{C}}^{2}\right) \\
& +\frac{1}{\Lambda_{-}^{6}}\left(4 \tilde{R}^{\mu \rho \alpha \nu} \nabla_{\rho} \nabla_{\nu} \mathcal{C}+4 R^{\mu \rho \alpha \nu} \nabla_{\rho} \nabla_{\nu} \tilde{\mathcal{C}}+\frac{g^{\mu \alpha}}{2} \tilde{\mathcal{C}} \mathcal{C}\right),
\end{aligned}
$$

and hold around a vacuum solution at order $\mathcal{O}\left(1 / \Lambda^{6}, 1 / \tilde{\Lambda}^{6}, 1 / \Lambda_{-}^{6}\right)$.

Spherically symmetric solutions.-From now on, we use dimensionless coupling parameters,

$$
\left(\epsilon_{1}, \epsilon_{2}, \epsilon_{3}\right)=\left(\frac{1}{M^{6} \Lambda^{6}}, \frac{1}{M^{6} \tilde{\Lambda}^{6}}, \frac{1}{M^{6} \Lambda_{-}^{6}}\right),
$$

where $M$ is the gravitational mass of the spacetime [12]. Consider spherically symmetric, static vacuum solutions:

$d s^{2}=-f_{t}^{\epsilon_{i}}(r) d t^{2}+\frac{1}{f_{r}^{\epsilon_{i}}(r)} d r^{2}+r^{2}\left(d \theta^{2}+\sin ^{2} \theta d \phi^{2}\right)$.

In this setup, $\tilde{\mathcal{C}}=0, \tilde{R}^{\mu \rho \alpha \nu} \nabla_{\rho} \nabla_{\nu} \mathcal{C}=0$. Since these are small corrections to GR, we look for slight deviations from the Schwarzschild geometry, $f_{t, r}^{\epsilon_{i}}=1-2 M / r+\epsilon_{i} \delta f_{t, r}^{\epsilon_{i}}$. Asymptotic flatness yields [13]

$$
\begin{gathered}
f_{t}^{\epsilon_{i}}=1-\frac{2 M}{r}+\epsilon_{i} \delta_{1}^{i}\left(-\frac{1024 M^{9}}{r^{9}}+\frac{1408 M^{10}}{r^{10}}\right), \\
f_{r}^{\epsilon_{i}}=1-\frac{2 M}{r}+\epsilon_{i} \delta_{1}^{i}\left(-\frac{4608 M^{9}}{r^{9}}+\frac{8576 M^{10}}{r^{10}}\right) .
\end{gathered}
$$

This spacetime describes a $\mathrm{BH}$, with an event horizon located at $r=r_{H}=2 M\left(1+5 \epsilon_{1} / 8\right)+\mathcal{O}\left(\epsilon_{i}^{2}\right)$. As expected (these are higher-curvature modifications), the corrections decay very fast with the radial distance $r$. The usual Parameterized Post-Newtonian parameters $\gamma, \beta$, e.g., [14], are the same as GR. Likewise, GWs in this theory propagate at the speed of light when far from sources and the dispersion relation is the same as GR. $\mathcal{O}\left(\epsilon_{1}^{2}\right)$ corrections are shown in the Supplemental Material [15], indicating that these solutions can be trusted for $\epsilon_{1} \lesssim 0.04$. However, one should keep in mind that the leading corrections are of order $\epsilon^{4 / 3} \sim \mathcal{O}\left(1 / \Lambda^{8}\right)$ from operators schematically of order $R_{\mu \nu \rho \sigma}^{5}$ in Eq. (1) [11].

Slowly spinning $B H s$ and $\mathbb{Z}_{2}$-symmetry breaking. -The geometry above can be extended to include angular momentum as

$$
\begin{aligned}
d s^{2}= & -f_{t}[1+2 h] d t^{2}+[1+2 m] \frac{d r^{2}}{f_{r}} \\
& +r^{2}[1+2 k]\left[d \theta^{2}+\sin ^{2} \theta(d \phi-\hat{\omega} d t)^{2}\right],
\end{aligned}
$$

where $f_{t}, f_{r}$ are the nonspinning metric functions Eqs. (5) and (6), and $(h, m, k, \hat{\omega})$ are functions of $r, \theta$ only, and perturbative in the angular momentum $J=M^{2} \chi$. The angular dependence can be expanded in terms of the Legendre polynomials, while the radial components are expressed as a series in powers of $\chi[17,18]$. Inserting the previous ansatz into the field equations (2) we can solve for the unknown metric functions $(h, m, k, \hat{\omega})$ order by order in the BH spin [19]. The full solution was computed up to $\mathcal{O}\left(\chi^{4}\right)$. The explicit form of the metric tensor and other quantities in this Letter can be found online [16].

Having derived the full metric it is straightforward to compute the main properties of the slowly spinning $\mathrm{BH}$ at the desired order in $J$. The horizon, e.g., is located at

$$
\frac{r_{H}}{M}=2+\frac{5 \epsilon_{1}}{4}-\chi^{2}\left(\frac{1}{4}+\frac{51 \epsilon_{1}}{352}-\frac{72 \epsilon_{2}}{11}\right) .
$$

Note that $r_{H}$ depends on even powers of $\chi$ only. Moreover, the spin terms turn on $\epsilon_{2}$ corrections which were absent for the static solutions. The equatorial frequency at the innermost stable circular orbit (ISCO) and at the photosphere are 


$$
\begin{aligned}
M \Omega_{\mathrm{ISCO}}= & \frac{1}{6 \sqrt{6}}-\frac{5291 \epsilon_{1}}{1889568 \sqrt{6}} \\
+ & \chi\left(\frac{11}{216}-\frac{2059367 \epsilon_{1}}{374134464}+\frac{211 \epsilon_{2}}{144342}\right), \\
M \Omega_{\mathrm{LR}}= & \frac{1}{3 \sqrt{3}}-\frac{832 \epsilon_{1}}{59049 \sqrt{3}} \\
& +\chi\left(\frac{2}{27}-\frac{159872 \epsilon_{1}}{5845851}+\frac{2048 \epsilon_{2}}{72171}\right) .
\end{aligned}
$$

Even though $\epsilon_{1} \sim 1$ induces changes in the horizon area of order $\mathcal{O}(1)$, this is not an observable. By contrast, the gauge-independent frequency $\Omega_{\text {ISCO }}$ suffers much smaller corrections even for such large couplings: a small increase in radial distance translates into a region of slightly smaller curvature and much less affected by such curvaturesensitive terms. This is also true for other relativistic effects associated with frame dragging. For example, the orbital plane of test particles, if not aligned with the equatorial plane, will precess around the angular momentum axis of the rotating body, the Lense-Thirring effect [20]. We can investigate this phenomenon studying the precession frequencies $\Omega_{r}$ and $\Omega_{\theta}$, which describe the perturbation in circular orbits due $r$ and $\theta$ velocity components [21,22] (analytic expressions for these quantities can be found online [16]).

The $\mathrm{BH}$ quadrupole moment is [23]

$$
Q_{20}=-\sqrt{\frac{64 \pi}{15}} \chi^{2}\left(1-\frac{89 \epsilon_{1}}{25}+\frac{216 \epsilon_{2}}{25}\right) .
$$

Observations probing only the background geometry, such as those using electromagnetic observations of matter close to the galactic center, can be used to constraint the coupling parameters $\epsilon_{i}$. Most of the constraints on alternatives to the Kerr geometry use ad hoc parametrizations $[1,2]$. The geometry above either does not fit into such parametrized metrics or, when it does, was never studied in the context of actual observations (see, e.g., Refs. [24,25]).

The $\epsilon_{3}$ parameter adds an interesting "twist" to the solutions, not apparent from equatorial observables. At linear order in rotation, such coupling introduces the following corrections to the background geometry:

$$
\begin{aligned}
\delta g_{\mu \nu} d x^{\mu} d x^{\nu}= & \epsilon_{3} \chi\left(\frac{73728 M^{9}}{r^{9}} d r^{2}+\frac{256 M^{9}(243 M-160 r)}{5 r^{8}}\right. \\
& \left.\times\left(d \theta^{2}+\sin ^{2} \theta d \phi^{2}\right)\right) \cos \theta
\end{aligned}
$$

This is the first purely gravitational example of a $\mathbb{Z}_{2}$-symmetry violating BH solution. (Recently, Ref. [26] reported a similar finding, but for theories with nonminimal couplings and extra fields.) The above form is not a coordinate artifact: curvature scalars at the horizon are indeed affected by it, e.g., $R=27 \chi \epsilon_{3} \cos \theta /\left(4 M^{2}\right)$, $\delta \mathcal{C}=189 \chi \epsilon_{3} \cos \theta /\left(8 M^{4}\right), \delta \tilde{\mathcal{C}}=0$, to linear order in spin. The linear stability of this solution is unclear at this stage.

Dynamics.-Consider now the dynamics of the spherically symmetric solution Eq. (4). Fourier decompose and expand the fluctuations in spherical tensor harmonics. The tensor harmonics are of odd ("-") and even ("+") type, depending on their transformation properties, and these different sectors usually decouple [27-29]. The form of the perturbed metric is

$$
h_{\mu \nu}^{(-)} d x^{\mu} d x^{\nu}=2 e^{-i \omega t} \sin \theta \partial_{\theta} Y_{\ell 0} d \phi\left(h_{0} d t+h_{1} d r\right)
$$

and

$$
\begin{aligned}
h_{\mu \nu}^{(+)} d x^{\mu} d x^{\nu}= & e^{-i \omega t} Y_{\ell 0}\left[f_{t} H_{0} d t^{2}+2 H_{1} d t d r\right. \\
& \left.+f_{r}^{-1} H_{2} d r^{2}+r^{2} K\left(d \theta^{2}+\sin ^{2} \theta d \phi^{2}\right)\right],
\end{aligned}
$$

respectively, where $h_{0}, h_{1}, H_{0}, H_{1}, H_{2}$, and $K$ are functions of $r$, and $Y_{\ell 0}$ is the spherical harmonics $Y_{\ell m=0}$.

Given the structure of the field equations, the equations of motion are described by up to fourth-order differential equations. However, (i) when the coupling parameters $\epsilon_{i}$ are taken to be perturbatively small, one can use the zerothorder GR equations to reduce the problem to second-order differential equations, and (ii) the odd and even parity perturbations around the spacetime Eq. (4) for the theory Eq. (1) are decoupled for $\epsilon_{1}$ and $\epsilon_{2}$ corrections but coupled for $\epsilon_{3}$ corrections, due to the parity violating terms.

To derive the perturbed field equations we replace the metric $g_{\mu \nu}+h_{\mu \nu}^{(-)}+h_{\mu \nu}^{(+)}$into Eq. (2) and expand the equations up to $\mathcal{O}\left(h_{\mu \nu}^{( \pm)}\right)$and $\mathcal{O}\left(\epsilon_{i}\right)$. In GR, the perturbed field equations around the Schwarzschild spacetime can be combined into single master functions,

$$
\begin{aligned}
& \Psi_{-}^{\mathrm{GR}}=\frac{i f h_{1}(r)}{r \omega}, \\
& \Psi_{+}^{\mathrm{GR}}=\frac{1}{\lambda r+6 M}\left(-r^{2} K+\frac{i f r H_{1}}{\omega}\right),
\end{aligned}
$$

and they satisfy the Regge-Wheeler and Zerilli equations,

$$
\frac{d^{2} \Psi_{ \pm}^{\mathrm{GR}}}{d r_{*}^{2}}+\left(\omega^{2}-f V_{ \pm}^{\mathrm{GR}}\right) \Psi_{ \pm}^{\mathrm{GR}}=0,
$$

with

$$
\begin{gathered}
V_{-}^{\mathrm{GR}}=\frac{(\lambda+2)}{r^{2}}-\frac{6 M}{r^{3}}, \\
V_{+}^{\mathrm{GR}}=\frac{1}{r^{3}(\lambda r+6 M)^{2}}\left[36 \lambda M^{2} r+6 \lambda^{2} M r^{2}\right. \\
\left.+\lambda^{2}(\lambda+2) r^{3}+72 M^{3}\right],
\end{gathered}
$$


where $\lambda=\ell^{2}+\ell-2, f=1-2 M / r$, and $d r / d r_{*}=f$. All components of $h_{\mu \nu}^{( \pm)}$, i.e., $h_{0}, h_{1}, H_{0}, H_{1}, H_{2}$, and $K$, are expressed by master variables $\Psi_{ \pm}^{\mathrm{GR}}$ at this lowest order.

Clearly, $\Psi_{ \pm}^{\epsilon_{i}}=\Psi_{ \pm}^{\mathrm{GR}}+\mathcal{O}\left(\epsilon_{i}\right)$. At linear order in $h_{\mu \nu}^{( \pm)}$, the right-hand side of Eq. (2) takes the form $\epsilon_{i} \times \mathcal{O}\left(h_{\mu \nu}^{( \pm)}\right)$. We can use the $\mathcal{O}\left(\epsilon_{i}^{0}\right)$ relations among $h_{\mu \nu}^{( \pm)}$and the master variables $\Psi_{ \pm}^{\epsilon_{i}}$ to compute $\mathcal{O}\left(h_{\mu \nu}^{( \pm)}\right)$terms in the right-hand side of Eq. (2), because $\mathcal{O}\left(\epsilon_{i}\right)$ corrections in $h_{\mu \nu}^{( \pm)}$become higher order in $\epsilon_{i}$. In this way, all the $h_{\mu \nu}^{( \pm)}$in the right-hand side of Eq. (2) can be replaced with $\Psi_{ \pm}^{\epsilon_{i}}$. At $\mathcal{O}\left(\epsilon_{i}^{0}\right), \Psi_{ \pm}^{\epsilon_{i}}$ satisfies Eq. (13); hence, by using Eq. (13) and its derivatives with respect to $r$, we can replace higher-order derivatives into lower derivatives. The above is a procedure to obtain second-order equations of motion at linear level. For an initial study on how to do this at nonlinear level, see, e.g., Ref. [30].

Tidal Love numbers. - When an object is placed in an external gravitational field, it is tidally deformed. Such effect can be quantified through the calculation of the object's tidal Love numbers (TLNs) [31-33]. BHs in GR possess the remarkable property that their TLNs are zero [32-37]. Nonzero TLNs are therefore a good discriminator of new physics [37-39]. We find the following quadrupolar TLNs for BHs in this theory, using the conventions of Ref. [37],

$$
\begin{gathered}
k_{2}^{E}=\frac{1008}{25} \epsilon_{1}, \quad k_{2}^{B}=\frac{3072}{175} \epsilon_{1}, \\
k_{2}^{E}=0, \quad k_{2}^{B}=-\frac{384}{5} \epsilon_{2},
\end{gathered}
$$

for polar and axial-type quadrupolar external fields, respectively. For $\epsilon_{3}$ corrections, we find that an external axial quadrupolar field induces a polar quadrupolar moment in the spacetime. New TLNs seem to be needed to describe this scenario, but we will not dwell on this any further.

Quasinormal modes and stability.-After the procedure above, the dynamics of slightly disturbed nonspinning BHs in this theory are described by very simple master equations, presented in the online Mathematica notebook [16]. It should be noted that the master equations are of second order in spatial and time derivatives, with no mixing. Higher-order terms cancel out. The master equations are completely decoupled for $\epsilon_{1}, \epsilon_{2}$. Computing the characteristic quasinormal frequencies is then a straightforward procedure [16,29]. Define, for each multipole $\ell$,

$$
\delta_{l} \equiv\left(\frac{\operatorname{Re}\left(\omega-\omega_{0}\right)}{\epsilon_{i} \operatorname{Re}\left(\omega_{0}\right)}, \frac{\operatorname{Im}\left(\omega-\omega_{0}\right)}{\epsilon_{i} \operatorname{Im}\left(\omega_{0}\right)}\right),
$$

where $\omega_{0}$ is the unperturbed GR value.

$\epsilon_{1}$ corrections.-We used a direct integration approach to compute the quasinormal modes of odd and even parity perturbations, and two different independent codes. We find

$$
\begin{array}{ll}
\delta_{2}^{\text {even }}=(0.45,-2.75), & \delta_{2}^{\text {odd }}=(0.22,-0.64), \\
\delta_{3}^{\text {even }}=(1.07,-6.42), & \delta_{3}^{\text {odd }}=-(0.0099,0.44), \\
\delta_{4}^{\text {even }}=(1.76,-11.43), & \delta_{4}^{\text {odd }}=-(0.048,0.19) .
\end{array}
$$

$\epsilon_{2}$ corrections.-For $\epsilon_{2}$ couplings, we find

$$
\begin{array}{ll}
\delta_{2}^{\text {even }}=0, & \delta_{2}^{\text {odd }}=(2.18,-15.85), \\
\delta_{3}^{\text {even }}=0, & \delta_{3}^{\text {odd }}=(4.23,-30.47), \\
\delta_{4}^{\text {even }}=0, & \delta_{4}^{\text {odd }}=(6.99,-49.54) .
\end{array}
$$

Note that $\tilde{\mathcal{C}}=\mathcal{O}\left[\left(h_{\mu \nu}^{(+)}\right)^{2}\right]$ for even perturbations which explains why its even sector is identical to GR. Notice also that even (odd) corrections scale as $\sim l^{2}$ for $\epsilon_{1}\left(\epsilon_{2}\right)$ couplings. In fact, at large $l$ there is an extrema in the potential at $r=11 M / 5$. Thus, these modes are localized away from the light ring and closer to the horizon. This introduces a new scale in the problem, and raises the interesting possibility that higher multipoles arrive later, producing a phenomena similar to echoes in the late-time waveform [9,10,40-43]. The results for $\epsilon_{3}$ corrections will be discussed elsewhere. At sufficiently small couplings (including most likely the regime of validity of the EFT), the quasinormal frequencies are only small corrections to the GR values, and the system is therefore stable. Notice that from a purely mathematical point of view, the structure of the master equations is such that the system is linearly mode stable for any positive $\epsilon_{i}$ (see Supplemental Material [15]).

Constraints on this theory can be obtained through a careful analysis of the post-Newtonian regime [11]. However, due to the strong dependence on the curvature, probes of the region close to the horizon-such as quasinormal modes-are clearly at an advantage. From the requirement that $\left(\omega-\omega_{0}\right) / \omega_{0} \lesssim 1$, we find that LIGO observations constrain $\left(1 / \Lambda, 1 / \tilde{\Lambda}, 1 / \Lambda_{-}\right) \sim 100 \mathrm{Km}$, from the first event GW150914 [44]. One can foresee improvements of 1 order of magnitude, but not more than that, from ringdown signals. The space-based detector LISA will be sensitive to lower frequencies, therefore larger masses, and so different $\Lambda$ 's [45] (see Ref. [11] for a discussion).

Discussion.-The search for signatures of deviations from Einstein's gravity is a challenging program. Theoretical bias may lead us to expect no changes at all, on scales which current detectors can probe. However, if one abandons such prejudice, the possibilities to change gravity are very large. Thus, it is customary to focus on possible generic smoking-gun effects, or then on a handful of theories which one knows well enough to calculate observables $[1,2]$. One appealing simple and generic class of theories consists of either minimally or weakly coupled 
light scalars [46]. Such a broad class of theories gives rise to clear signatures, observable in both GW detectors and traditional telescopes [47-51]. In the absence of scalar degrees of freedom, EFT arguments suggest that Eq. (1) describes the dominant effects of the most generic class of theories compatible with reasonable requirements [11].

Our result for the curvature corrected geometry of nonspinning geometries, Eqs. (5) and (6), shows why it is difficult to probe such corrections: they decay extremely fast at large distances, and even at the light ring they are suppressed (in fact, such result generalizes easily to higher-order theories, as shown in the Supplemental Material [15]). The observational prospects appear to be much more promising for the signal associated with the quasinormal modes.

The results we derived are of interest for GW detectors, but also to VLBI observatories such as GRAVITY [52] or the Event Horizon telescope [53,54], providing a physically motivated and understood geometry with which to perform tests of GR.

L.S. would like to thank Junwu Huang and Victor Gorbenko for collaboration in the very early stages of this work. We would like to thank Leonardo Gualtieri for useful comments on an earlier version of this manuscript. V. C. and L. S. would like to thank ICTP for hospitality where part of the work was initiated during the "Summer School on Cosmology." The authors acknowledge financial support provided under the European Union's H2020 ERC Consolidator Grant "Matter and strong-field gravity: New frontiers in Einstein's theory" Grant Agreement No. MaGRaTh-646597. This project has received funding from the European Union's Horizon 2020 research and innovation programme under the Marie Sklodowska-Curie Grant Agreement No. 690904. The authors would like to acknowledge networking support by the GWverse COST Action CA16104, "Black holes, gravitational waves and fundamental physics." L. S. is partially supported by Simons Foundation Origins of the Universe program (Modern Inflationary Cosmology collaboration) and by NSF Grant No. 1720397. Computations were performed on the "Baltasar Sete-Sois" cluster at IST.

[1] L. Barack et al., arXiv:1806.05195.

[2] E. Berti et al., Classical Quantum Gravity 32, 243001 (2015).

[3] V. Cardoso and L. Gualtieri, Classical Quantum Gravity 33, 174001 (2016).

[4] C. A. R. Herdeiro and E. Radu, Int. J. Mod. Phys. D 24, 1542014 (2015).

[5] T. P. Sotiriou, Classical Quantum Gravity 32, 214002 (2015).

[6] E. Berti, V. Cardoso, and C. M. Will, Phys. Rev. D 73, 064030 (2006).

[7] E. Berti, A. Sesana, E. Barausse, V. Cardoso, and K. Belczynski, Phys. Rev. Lett. 117, 101102 (2016).
[8] H. Yang, K. Yagi, J. Blackman, L. Lehner, V. Paschalidis, F. Pretorius, and N. Yunes, Phys. Rev. Lett. 118, 161101 (2017).

[9] V. Cardoso and P. Pani, Nat. Astron. 1, 586 (2017).

[10] V. Cardoso and P. Pani, arXiv:1707.03021.

[11] S. Endlich, V. Gorbenko, J. Huang, and L. Senatore, J. High Energy Phys. 09 (2017) 122.

[12] All EFTs have some numerical ambiguity in defining the expansion parameter. The actual definition is inconsequential for the correctness of the calculation, but it indicates the range of validity of the framework and the actual size of the corrections. With our choice, the corrections to the metric are of order $\epsilon$ at $r \sim r_{H}$, a reasonable outcome. A more sensible definition would consider the magnitude of the next-order correction, but we leave this improved definition of $\epsilon$ to future work.

[13] The seemingly large coefficients in the metric corrections are only artifacts of our choice of parametrization: Had we chosen $r_{h}$ instead of the mass $m$, all coefficients would be of order unity.

[14] C. M. Will, Living Rev. Relativity 9, 3 (2006).

[15] See Supplemental Material at http://link.aps.org/ supplemental/10.1103/PhysRevLett.121.251105 for details on how to derive the master equations for gravitational perturbations, higher order corrections to the spherically symmetric solution, and a generalization to higher-order theories. The online notebooks [16] provide all the equations for the metric, the perturbations, and the geodesic quantities.

[16] Gravitation in técnico: files, https://centra.tecnico.ulisboa .pt/network/grit/files/, accessed August 2018.

[17] J. B. Hartle, Astrophys. J. 150, 1005 (1967).

[18] J. B. Hartle and K. S. Thorne, Astrophys. J. 153, 807 (1968).

[19] A. Maselli, P. Pani, L. Gualtieri, and V. Ferrari, Phys. Rev. D 92, 083014 (2015).

[20] B. Mashhoon, F. W. Hehl, and D. S. Theiss, Gen. Relativ. Gravit. 16, 711 (1984).

[21] L. Stella and M. Vietri, Astrophys. J. Lett. 492, L59 (1998).

[22] A. Maselli, P. Pani, R. Cotesta, L. Gualtieri, V. Ferrari, and L. Stella, Astrophys. J. 843, 25 (2017).

[23] K. S. Thorne, Rev. Mod. Phys. 52, 299 (1980).

[24] T. Johannsen, A. E. Broderick, P. M. Plewa, S. Chatzopoulos, S. S. Doeleman, F. Eisenhauer, V. L. Fish, R. Genzel, O. Gerhard, and M. D. Johnson, Phys. Rev. Lett. 116, 031101 (2016).

[25] T. Johannsen, C. Wang, A. E. Broderick, S. S. Doeleman, V. L. Fish, A. Loeb, and D. Psaltis, Phys. Rev. Lett. 117, 091101 (2016).

[26] P. V. P. Cunha, C. A. R. Herdeiro, and E. Radu, arXiv: 1808.06692 .

[27] T. Regge and J. A. Wheeler, Phys. Rev. 108, 1063 (1957).

[28] F. Zerilli, Phys. Rev. D 2, 2141 (1970).

[29] E. Berti, V. Cardoso, and A. O. Starinets, Classical Quantum Gravity 26, 163001 (2009).

[30] G. Allwright and L. Lehner, arXiv:1808.07897.

[31] E. E. Flanagan and T. Hinderer, Phys. Rev. D 77, 021502 (2008).

[32] T. Binnington and E. Poisson, Phys. Rev. D 80, 084018 (2009). 
[33] T. Damour and A. Nagar, Phys. Rev. D 80, 084035 (2009).

[34] R. A. Porto, Phys. Rep. 633, 1 (2016).

[35] R. A. Porto, Fortschr. Phys. 64, 723 (2016).

[36] I. Rothstein, Analytic Calculations of Gravitational Wave Signatures During Early Stages of Inspirals, in Proceedings at the Simons Center Meeting "GW161212: The Universe through gravitational waves," 2017.

[37] V. Cardoso, E. Franzin, A. Maselli, P. Pani, and G. Raposo, Phys. Rev. D 95, 084014 (2017); 95, 089901(E) (2017).

[38] B. Kol and M. Smolkin, J. High Energy Phys. 02 (2012) 010 .

[39] A. Maselli, P. Pani, V. Cardoso, T. Abdelsalhin, L. Gualtieri, and V. Ferrari, Phys. Rev. Lett. 120, 081101 (2018).

[40] V. Cardoso, E. Franzin, and P. Pani, Phys. Rev. Lett. 116, 171101 (2016); 117, 089902(E) (2016).

[41] J. Abedi, H. Dykaar, and N. Afshordi, Phys. Rev. D 96, 082004 (2017).

[42] Z. Mark, A. Zimmerman, S. M. Du, and Y. Chen, Phys. Rev. D 96, 084002 (2017).

[43] M. R. Correia and V. Cardoso, Phys. Rev. D 97, 084030 (2018).
[44] B. P. Abbott et al. (LIGO Scientific and Virgo Collaborations), Phys. Rev. Lett. 116, 221101 (2016).

[45] H. Audley et al. (LISA Collaboration), arXiv:1702.00786.

[46] A. Arvanitaki, S. Dimopoulos, S. Dubovsky, N. Kaloper, and J. March-Russell, Phys. Rev. D 81, 123530 (2010).

[47] R. Brito, V. Cardoso, and P. Pani, Classical Quantum Gravity 32, 134001 (2015).

[48] A. Arvanitaki, M. Baryakhtar, S. Dimopoulos, S. Dubovsky, and R. Lasenby, Phys. Rev. D 95, 043001 (2017).

[49] R. Brito, S. Ghosh, E. Barausse, E. Berti, V. Cardoso, I. Dvorkin, A. Klein, and P. Pani, Phys. Rev. D 96, 064050 (2017).

[50] M. C. Ferreira, C. F. B. Macedo, and V. Cardoso, Phys. Rev. D 96, 083017 (2017).

[51] M. Boskovic, F. Duque, M. C. Ferreira, F. S. Miguel, and V. Cardoso, Phys. Rev. D 98, 024037 (2018).

[52] R. Abuter et al. (GRAVITY Collaboration), Astron. Astrophys. 615, L15 (2018).

[53] D. Psaltis, arXiv:1806.09740.

[54] H. Falcke and S. B. Markoff, Classical Quantum Gravity 30, 244003 (2013). 